\title{
Etude diagnostique de la flore ornementale togolaise
}

\author{
Raoufou RADJI*, Kouami KOKOU et Koffi AKPAGANA \\ Laboratoire de Botanique et Ecologie Végétale, Faculté des Sciences, Université de Lomé, BP : 1515, \\ Lomé, TOGO \\ *Auteur correspondant, E-mail : pradji@ hotmail.com/pradji@tg.refer.org, Tel : (+228) 225-50-94, \\ Fax: (+228) 221-85-95
}

\section{RESUME}

L'horticulture ornementale est en plein essor au Togo, mais reste méconnue, ainsi que son secteur commercial. Cette étude réalise un inventaire floristique et présente la morphologie des espèces utilisées en horticulture ornementale au Togo. Sur les 55 exploitations étudiées, 612 espèces sont recensées et identifiées. Ces espèces sont regroupées en 72 familles et les plus riches en nombre de genre sont entre autres les Arecaceae et les Cactaceae. Par ailleurs, les plus visibles dans le pays sont les Nyctaginaceae, les Annonaceae et les Rubiaceae. Très peu de ces espèces sont autochtones pourtant, il est urgent de mieux valoriser la flore locale spontanée.

(C) 2010 International Formulae Group. All rights reserved.

Mots-clés : Horticulture urbaine, pépinières, biodiversité, valorisation, flore locale.

\section{INTRODUCTION}

Actuellement, la flore du Togo est estimée à 3451 espèces dont 509 sont introduites (Akpagana, 1992b ; Guelly, 1994 ; Kokou, 1998). Bien que plusieurs exploitations ou pépinières de plantes ornementales s'observent à tous les coins de rues à Lomé et de plus en plus dans les villes secondaires du pays, ces estimations font très peu mention des espèces abondamment utilisées en horticulture urbaine (Brock et Foeken, 2006; Fleury et al., 2008). Cette activité emploie des centaines de jeunes et, prolifère certainement à cause de sa rentabilité économique, contribuant ainsi à la résorption du taux de chômage (Aké Assi, 2002 ; Radji, 1998). Ce secteur informel n'a pas le niveau d'organisation et le professionnalisme qu'on connaît dans certains pays du continent comme le Kenya (Jaffee et Masakure, 2005 ; Neven et al., 2009) ou ailleurs dans d'autres pays en développement qui exportent des quantités importantes de fleurs dans le monde (Roy et Thorat, 2007). L'expansion de l'horticulture urbaine à Lomé et dans d'autres villes du Togo, accroît le nombre de taxons et mobilise une importante phytobiodiversité constituée aussi bien de la flore autochtone que d'espèces introduites depuis très longtemps ou récemment. Le plus souvent, la composition floristique des pépinières ou des exploitations horticoles et les bénéfices que les acteurs peuvent en tirer, sont méconnues tout comme les risques d'envahissement (Maki et Galatowitsch, 2004). 
C'est dans ce cadre que la présente étude se propose d'élaborer un état des lieux qui devra servir à l'élaboration de stratégies de conservation et de gestion durable de la biodiversité horticole au Togo. Il s'agit de réaliser un inventaire botanique le plus exhaustif possible des espèces végétales utilisées en horticulture urbaine au Togo et, de présenter quelques unes de leurs caractéristiques morphologique et phytogéographique. Les enjeux d'un tel diagnostic sont d'une part, une meilleure valorisation et d'autre part, mettre fin à la spéculation parfois fâcheuse (vente d'une plante à la place d'une autre) et limiter ainsi les risques d'intoxication car certaines plantes sont utilisées en pharmacopée traditionnelle.

\section{MATERIEL ET METHODES}

Un recensement floristique exhaustif est réalisé dans toutes les exploitations horticoles pépinières, parcs et jardins répartis sur le territoire togolais. Au niveau de chaque site, un relevé est effectué quel que soit sa surface. Cet inventaire a permis de connaître la surface moyenne des exploitations et de dresser un répertoire complet des espèces horticoles du Togo. L'identification scientifique des espèces récoltées est assurée à l'aide des flores de Berhaut (1971 à 1988), de Byrd Graf (1981), de Houérou (Le) et al. (1987), de Grisvard et al. (1990), de Hessayon (1992) et de Don Ellison (1995). D’autres informations sont obtenues avec celles de Hutchison et Dalziel (1954-1972), de Brunel et al. (1984), et de Lebrun et Stork (2003, 2006, 2008). Les noms d'auteurs et les synonymies proviennent des données du code international de la nomenclature botanique (2007), du site web de la Base de données des plantes d'Afrique du Jardin Botanique de Génève et des sites web de l'Index Nominum Genericorum (ING) et de l'International Plant Names Index (IPNI). Les échantillons récoltés ont été déposés dans l'herbier de l'Université de Lomé. La nomenclature suit Lebrun et Stork (2008). Les auteurs des noms scientifiques sont mentionnés dans le Tableau 1. L'aire basale de chaque station est mesurée à l'aide d'un décamètre.
Les informations extraites de cette liste sont diverses. Il s'agit entre autres de l'importance des types biologiques et morphologiques (Raunkier, 1934 ; Richards et al., 1940), de la diversité botanique, de l'importance des espèces caractéristiques des pépinières (espèces fréquentes, rares, etc.) et de leur chorologie. La richesse spécifique est exprimée par le nombre total d'espèces observées (en valeur absolue ou par unité de surface). L'indice de Shannon-Wiener est obtenu à l'aide de la formule $\mathrm{H}^{\prime}=-\Sigma \mathrm{ni} / \mathrm{N} \log 2$ $(n i / N)$ où ni est l'effectif de l'espèce $\mathrm{i}$ et $\mathrm{N}$ l'effectif total des espèces (Fongnzossie et al., 2008). L'indice de Simpson a servi à mesurer la probabilité pour que deux individus choisis au hasard dans une population infinie, appartiennent à la même espèce. Il est exprimé de l'équation $\mathrm{D}^{\prime}=\Sigma(\mathrm{ni} / \mathrm{N}) / 2$.

L'équitabilité de Piélou, exprime le rapport entre la diversité observée et la diversité maximale et permet de rendre compte de la distribution des espèces dans l'échantillon. Il est donné par la formule Eq= $\mathrm{H}^{\prime} / \log 2 \mathrm{~N}$.

Le «species rarity-weighted richness index ", indique le statut des espèces. Il a été calculé suivant l'équation RI = $(1-(\mathrm{ni} / \mathrm{N}))$ $\mathrm{X} 100$; avec RI comme indice de raréfaction, ni : nombre de relevés dans lequel l'espèce $\mathrm{i}$ est présent et $\mathrm{N}$ le nombre total de relevés. Conformément à cette relation, les espèces dont $\mathrm{RI}<80 \%$ sont considérées comme des espèces préférentielles très fréquentes dans les pépinières étudiées. Celles dont RI $>80 \%$ sont rares. Pour chaque espèce, le type morphologique est pris en compte.

\section{RESULTATS}

L'aire moyenne des stations horticoles prospectées est de $288 \pm 202 \mathrm{~m}^{2}$. L'écart-type indique la grande variabilité entre les surfaces. Dans les 55 stations, 612 espèces ont été recensées (Tableau 1). Ces espèces se répartissent en 246 genres et 72 familles. Elles sont surtout concentrées à Lomé où l'activité est plus développée avec $96 \%$ des espèces contre $4 \%$ seulement pour les villes Atakpamé et Kpalimé. La culture de plantes ornementales n'est pas pratiquée dans les autres villes du Togo (Tableau 2). 
Tableau 1 : Index alphabétique des espèces ornementales rencontrées au Togo.

\begin{tabular}{|c|c|}
\hline Familles & $\begin{array}{l}\text { Noms scientifiques des } \\
\text { taxons }\end{array}$ \\
\hline Mimosaceae & $\begin{array}{l}\text { Acacia auriculaeformis } \\
\text { A.Cunn. ex Benth. }\end{array}$ \\
\hline Mimosaceae & Acacia biflora R.Br. \\
\hline Euphorbiaceae & Acalypha hispida Burm.f. \\
\hline Euphorbiaceae & $\begin{array}{l}\text { Acalypha wilkesiana Müll. } \\
\text { Arg. }\end{array}$ \\
\hline Euphorbiaceae & $\begin{array}{l}\text { Acalypha wilkesiana Müll. } \\
\text { Arg. var. 'java white' }\end{array}$ \\
\hline Euphorbiaceae & $\begin{array}{l}\text { Acalypha wilkesiana Müll. } \\
\text { Arg. var. hoffmanii }\end{array}$ \\
\hline Euphorbiaceae & $\begin{array}{l}\text { Acalypha wilkesiana Müll. } \\
\text { Arg. var. macrophylla }\end{array}$ \\
\hline Euphorbiaceae & $\begin{array}{l}\text { Acalypha wilkesiana Müll. } \\
\text { Arg. var. moorea }\end{array}$ \\
\hline Euphorbiaceae & $\begin{array}{l}\text { Acalypha wilkesiana Müll. } \\
\text { Arg. var. tricolor }\end{array}$ \\
\hline Cactaceae & $\begin{array}{l}\text { Acanthocereus pentagonus } \\
\text { Britton \& Rose }\end{array}$ \\
\hline Acanthaceae & Acanthus mollis L. \\
\hline Acanthaceae & $\begin{array}{l}\text { Acanthus pubescens (Thomson } \\
\text { ex Oliv.) Engl. }\end{array}$ \\
\hline Apocynaceae & $\begin{array}{l}\text { Adenium obesum (Forssk.) } \\
\text { Roem. \& Schult. }\end{array}$ \\
\hline Bromeliaceae & $\begin{array}{l}\text { Aechmea caudata Lindman } \\
\text { variegata }\end{array}$ \\
\hline Bromeliaceae & Aechmea chantinii Bak. \\
\hline Bromeliaceae & Aechmea fasciata Bak. \\
\hline Agavaveae & Agave americana $\mathrm{L}$. \\
\hline Agavaveae & $\begin{array}{l}\text { Agave americana L. striata } \\
\text { Trel. }\end{array}$ \\
\hline Agavaveae & $\begin{array}{l}\text { Agave angustifolia Haw. } \\
\text { var.marginata }\end{array}$ \\
\hline Agavaveae & Agave filifera Salm-Dyck. \\
\hline Agavaveae & $\begin{array}{l}\text { Agave victoriae-reginae } \\
\text { Moore. }\end{array}$ \\
\hline Araceae & $\begin{array}{l}\text { Aglaonema commutatum } \\
\text { Schott. }\end{array}$ \\
\hline Araceae & $\begin{array}{l}\text { Aglaonema marantifolium } \\
\text { Blume }\end{array}$ \\
\hline Araceae & $\begin{array}{l}\text { Aglaonema modestum Schott } \\
\text { ex Engl. }\end{array}$ \\
\hline Araceae & $\begin{array}{l}\text { Aglaonema pseudo- } \\
\text { bracteatum Schott. }\end{array}$ \\
\hline
\end{tabular}

Apocynaceae

Apocynaceae

Apocynaceae

Araceae

Araceae

Araceae

Araceae

Araceae

Liliaceae

Liliaceae

Liliaceae

Liliaceae

Liliaceae

Liliaceae

Liliaceae

Zingiberaceae

Zingiberaceae

Zingiberaceae

Amaranthaceae

Amaranthaceae

Amaranthaceae

Amaranthaceae

Amaranthaceae

Amaranthaceae

Amaranthaceae

Bromeliaceae

Bromeliaceae

Asteraceae

Araceae

Araceae
Allamanda cathartica L. var. hendersonii Hort.

Allamanda neriifolia Hook.

Allamanda violacea Gardn.

Alocasia korthalsii Schott

Alocasia lowii Hook. f. grandis

Alocasia macrorhiza Schott

Alocasia plumbea Van Houtte

Alocasia sanderiana Bull.

Aloe aristata Haw.

Aloe brevifolia Mill.

Aloe eru Berger

Aloe eru Berger maculata

Aloe humilis Mill.

Aloe vera (L.) Burm.f.

Aloe vera L. chinensis

Alpinia purpurata (Vieill.)

K.Schum.

Alpinia sanderae Hort. Sand.

Alpinia zerumbet (Pers.)

B.L.Burtt \& R.M. Sm.

Variegata

Alternanthera bettzickiana

(Regel) G. Nicholson

Alternanthera bettzickiana

(Regel) G. Nicholson var.

aurea

Alternanthera dentata

(Moench) Scheygr.var.'Ruby'

Amaranthus cristata Noronha

Amaranthus hybridus L. subsp. cruentus (L.) Thell.

Amaranthus paniculatus L.

Amaranthus tricolor $\mathrm{L}$.

Ananas bracteatusSchult.f. striatus M.B.Foster

Ananas comosus (L.) Merr.

'Porteanus'

Andryala pinnatifida Aiton

Anthurium andraeanum Lind. Anthurium andraeanum Lind. var. rhodochlorum 
Araceae

Araceae

Araceae

Araceae

Portulacaceae

Acanthaceae

Ä̈zoaceae

Orchidaceae

Araliaceae

Araliaceae

Araucariaceae

Araucariaceae

Arecaceae

Aristolochiaceae

Aristolochiaceae

Aristolochiaceae

Aristolochiaceae

Liliaceae

Moraceae

Asclepiadaceae

Liliaceae

Liliaceae

Liliaceae

Liliaceae

Aspleniaceae

Aspleniaceae

Aspleniaceae

Aspleniaceae

Meliaceae

Poaceae

Poaceae
Anthurium andraeanum Lind. var. rubrum

Anthurium clarinervium

Schott.

Anthurium hookeri Kunth.

Anthurium scherzerianum

Schott.

Antigonon leptopus Hook.\&

Arn.

Aphelandra squarrosa Nees. Aptenia cordifolia Schwant. Arachnis flos-aeris (L.) Rchb. f.

Aralia elata Seem.

Aralia elata Seem. variegata

Araucaria cunninghamii Ait.

Araucaria excelsa R. Br.

Areca triandra Roxb.

Aristolochia brasiliensis Mart. \& Zucc.

Aristolochia elegans Mast.

Aristolochia fimbriata Cham.

Aristolochia littoralis D.

Parodi

Arthropodium cirrhatum R.Br.

Artocarpus nobilis J.R. \& G.

Forst.

Asclepias curassavica L.

Asparagus densiflorus (Kunth)

Jessop sprengeri

Asparagus plumosus Bak.

Asparagus plumosus Bak. var. compactus

Asparagus plumosus Bak. var. nanus

Asplenium bulbiferum Forst.

Asplenium mayii ht.May;

Gard.

Asplenium nidus L.

Asplenium viviparum (L.fil.)

C.Presl

Azadirachta indica A. Juss.

Bambusa multiplex (Lour.)

Raeusch. ex Schult. \&

Schult.f.

Bambusa nana Roxb.
Poaceae

Acanthaceae

Begoniaceae

Begoniaceae

Begoniaceae

Begoniaceae

Begoniaceae

Begoniaceae

Begoniaceae

Begoniaceae

Begoniaceae

Begoniaceae

Begoniaceae

Begoniaceae

Acanthaceae

Bromeliaceae

Arecaceae

Arecaceae

Nyctagynaceae

Nyctagynaceae

Nyctagynaceae

Nyctagynaceae

Nyctagynaceae

Nyctagynaceae

Nyctagynaceae

Nyctagynaceae

Nyctagynaceae

Nyctagynaceae

Arecaceae

Euphorbiaceae

Euphorbiaceae
Bambusa vulgaris Schrader ex

Wendl.

Barleria lupulina Lindl.

Begonia cathayana Hemsl.

Begonia cleopatra Lem.

Begonia decora Stapf.

rhizomatous

Begonia deliciosa Linden ex

Fotsch

Begonia exotica Lem.

Begonia masoniana Irmsch.

Begonia robusta Blume.

Begonia scandens Sw.

Begonia semperflorens Link et Otto.

Begonia venosa Skan.

Begonia x erythrophylla

Neumann

Begonia zebrina Hort.

Beloperone guttata Brandegee

Billbergia zebrina Lindl.

Bismarckia nobilis Hildebr. \&

H.Wendl.

Borassus aethiopum Mart.

Bougainvillea glabra Choisy

Bougainvillea harisii Hort.

Bougainvillea spectabilis

Willd.

Bougainvillea spectabilis

Willd. var. 'Mary Palmer

Bougainvillea spectabilis

Willd. var. rubra plena

Bougainvillea spectabilis

Willd. var. variegata

Bougainvillea spectabilis

Willd. var.alba plena

Bougainvillea $x$ buttiana Holtt. var. Mrs. McLean

Bougainvillea $x$ buttiana Holtt. var. praetoria

Bougainvillea $x$ buttiana

Holttum \& Standl.

Brahea armata Wats.

Breynia disticha J.R. \& G.

Forst.

Breynia nivosa Small. 


\begin{tabular}{|c|c|c|c|}
\hline $\begin{array}{l}\text { Caesalpiniaceae } \\
\text { Araceae }\end{array}$ & $\begin{array}{l}\text { Caesalpinia pulcherrima Sw. } \\
\text { Caladium bicolor Vent. }\end{array}$ & Cannaceae & $\begin{array}{l}\text { Canna generalis L.H.Bailey } \\
\text { 'Richard Wallace' }\end{array}$ \\
\hline Araceae & $\begin{array}{l}\text { Caladium hortulanum Birdsey } \\
\text { 'Citation' }\end{array}$ & Cannaceae & $\begin{array}{l}\text { Canna generalis L.H.Bailey } \\
\text { striatus }\end{array}$ \\
\hline Araceae & $\begin{array}{l}\text { Caladium hortulanum Birdsey } \\
\text { 'Debutante' } \\
\text { Caladium hortulanum Birdsey }\end{array}$ & Asclepiadaceae & $\begin{array}{l}\text { Canna indica } L . \\
\text { Caralluma } \\
\text { praegracilisOberm. }\end{array}$ \\
\hline Araceae & $\begin{array}{l}\text { 'Gen. W.B. Halderman' } \\
\text { 'Gadium hortulanum Birdsey }\end{array}$ & Caricaceae & Carica papaya $\mathrm{L}$. \\
\hline Araceae & $\begin{array}{l}\text { Caladium hortulanum Birdsey } \\
\text { 'John Peed' }\end{array}$ & $\begin{array}{l}\text { Arecaceae } \\
\text { Caesalpiniaceae }\end{array}$ & $\begin{array}{l}\text { Caryota mitis Lour. } \\
\text { Cassia alata } \mathrm{L} .\end{array}$ \\
\hline Araceae & $\begin{array}{l}\text { Caladium hortulanum Birdsey } \\
\text { 'Maid of Orleans' } \\
\text { Caladium hortulanum Birdsey }\end{array}$ & $\begin{array}{l}\text { Caesalpiniaceae } \\
\text { Casuarinaceae }\end{array}$ & $\begin{array}{l}\text { Cassia siamea Lam. } \\
\text { Casuarina equisetifolia } \mathrm{L} .\end{array}$ \\
\hline Araceae & $\begin{array}{l}\text { Caladium hortulanum Birdsey } \\
\text { 'Triomphe de l'Exposition' } \\
\text { Calathea albicans A.Brongn. }\end{array}$ & Apocynaceae & $\begin{array}{l}\text { Catharanthus roseus (L.) G. } \\
\text { Don }\end{array}$ \\
\hline Maranthaceae & ex Petersen & Amaranthaceae & Celosia argentea $\mathrm{L}$. \\
\hline Maranthaceae & $\begin{array}{l}\text { Calathea bella (W. Bull.) } \\
\text { Regel }\end{array}$ & Amaranthaceae & $\begin{array}{l}\text { Celosia argentea L. var. } \\
\text { pyramidalis }\end{array}$ \\
\hline Maranthaceae & $\begin{array}{l}\text { Calathea grandiflora } \mathrm{K} . \\
\text { Schum. }\end{array}$ & $\begin{array}{l}\text { Amaranthaceae } \\
\text { Asteraceae }\end{array}$ & $\begin{array}{l}\text { Celosia cristata } \mathrm{L} . \\
\text { Centratherum punctatum Cass. }\end{array}$ \\
\hline Maranthaceae & $\begin{array}{l}\text { Calathea makoyana E.Morr. \& } \\
\text { Boom }\end{array}$ & $\begin{array}{l}\text { Cactaceae } \\
\text { Cactaceae }\end{array}$ & Cereus aethiops \\
\hline Maranthaceae & $\begin{array}{l}\text { Calathea ornata Koern. } \\
\text { Calathea ornata Koern. roseo- } \\
\text { lineata Petersen }\end{array}$ & $\begin{array}{l}\text { Cactaceae } \\
\text { Cactaceae } \\
\text { Cactaceae }\end{array}$ & $\begin{array}{l}\text { Cereus hexagonus (L.) Mill. } \\
\text { Cereus peruvianus (L.) Mill. } \\
\text { Cereus tortuosus Forbes }\end{array}$ \\
\hline Maranthaceae & $\begin{array}{l}\text { Calathea ornata Koern. roseo- } \\
\text { picta }\end{array}$ & $\begin{array}{l}\text { Solanaceae } \\
\text { Solanaceae }\end{array}$ & $\begin{array}{l}\text { Cestrum diurnum } \mathrm{L} . \\
\text { Cestrum nocturnum } \mathrm{L} .\end{array}$ \\
\hline Maranthaceae & $\begin{array}{l}\text { Calathea ornata Koern. } \\
\text { sanderiana }\end{array}$ & $\begin{array}{l}\text { Arecaceae } \\
\text { Arecaceae }\end{array}$ & $\begin{array}{l}\text { Chamaedorea elegans Mart. } \\
\text { Chamaerops humilis L. }\end{array}$ \\
\hline Maranthaceae & $\begin{array}{l}\text { Calathea stromata Hort } \\
\text { Calathea zebrina (Sims.) }\end{array}$ & Liliaceae & $\begin{array}{l}\text { Chlorophytum bichetii Hort. } \\
\text { ex Backer }\end{array}$ \\
\hline Cuppressaceae & $\begin{array}{l}\text { Lindl. } \\
\text { Callitris intratropica R.T. } \\
\text { Baker \& H.G. Sim. }\end{array}$ & Liliaceae & $\begin{array}{l}\text { Chlorophytum comosum } \\
\text { (Thunb.) Jacques } \\
\text { Chlorophytum comosum }\end{array}$ \\
\hline Asclepiadaceae & $\begin{array}{l}\text { Calotropis procera (Aiton) } \\
\text { W.T.Aiton }\end{array}$ & Liliaceae & $\begin{array}{l}\text { (Thunb.) Jacques var. } \\
\text { variegatum }\end{array}$ \\
\hline Annonaceae & $\begin{array}{l}\text { Cananga odorata (Lam.) } \\
\text { Hook. f. \& Thoms. }\end{array}$ & Liliaceae & $\begin{array}{l}\text { Chlorophytum comosum } \\
\text { (Thunb.) Jacques var. vittatum }\end{array}$ \\
\hline Cannaceae & $\begin{array}{l}\text { Canna generalis L.H.Bailey } \\
\text { 'Brilliant' }\end{array}$ & Liliaceae & $\begin{array}{l}\text { Chlorophytum laxum } \mathrm{R} . \mathrm{Br} . \\
\text { Chrysalidocarpus lutescens }\end{array}$ \\
\hline Cannaceae & $\begin{array}{l}\text { Canna generalis L.H.Bailey } \\
\text { 'Cleopatra' }\end{array}$ & $\begin{array}{l}\text { Arecaceae } \\
\text { Asteraceae }\end{array}$ & Chrysanthemum frutescens $\mathrm{L}$. \\
\hline Cannaceae & $\begin{array}{l}\text { Canna generalis L.H.Bailey } \\
\text { 'Confetti' }\end{array}$ & Poaceae & $\begin{array}{l}\text { Chrysopogon aciculatus } \\
\text { (Retz.) Trin. }\end{array}$ \\
\hline Cannaceae & $\begin{array}{l}\text { Canna generalls L.H.Ba1ley } \\
\text { 'Lucifer' }\end{array}$ & Rutaceae & Citrus aurantium $\mathrm{L}$. \\
\hline Cannaceae & $\begin{array}{l}\text { Canna generalis L.H.Bailey } \\
\text { 'President' }\end{array}$ & $\begin{array}{l}\text { Rutaceae } \\
\text { Rutaceae }\end{array}$ & $\begin{array}{l}\text { Citrus limon (L.) Burm. f. } \\
\text { Citrus maxima Merr. }\end{array}$ \\
\hline
\end{tabular}




\begin{tabular}{|c|c|c|c|}
\hline Rutaceae & Citrus nobilis Lour. & Funho & Codiaeum variegatum (L.) \\
\hline Rutaceae & Citrus sinensis Osbeck & выасеае & A.Juss. var.Mortimer \\
\hline Verbenaceae & $\begin{array}{l}\text { Clerodendrum fragrans Hort. } \\
\text { ex Vent. }\end{array}$ & Euphorbiaceae & $\begin{array}{l}\text { Codiaeum variegatum (L.) } \\
\text { A.Juss. var.rubrum }\end{array}$ \\
\hline Verbenaceae & Clerodendrum inerme Wall. & Euphorbiaceae & $\begin{array}{l}\text { Codiaeum variegatum (L.) } \\
\text { A.Juss. var. gloriosum }\end{array}$ \\
\hline Verbenaceae & $\begin{array}{l}\text { Clerodendrum speciosissimum } \\
\text { C. Morren }\end{array}$ & Lamiaceae & Coleus blumei Benth. \\
\hline Verbenaceae & $\begin{array}{l}\text { Clerodendrum thomsonae } \\
\text { Balf. }\end{array}$ & Lamiaceae & $\begin{array}{l}\text { Coleus blumei Benth. var. } \\
\text { 'brilliancy' }\end{array}$ \\
\hline Verbenaceae & $\begin{array}{l}\text { Clerodendrum x speciosum } \\
\text { Gürke }\end{array}$ & Lamiaceae & $\begin{array}{l}\text { Coleus blumei Benth. var. } \\
\text { 'klondyke' }\end{array}$ \\
\hline Fabaceae & Clitoria ternatea $\mathrm{L}$. & Lamiaceae & $\begin{array}{l}\text { Coleus blumei Benth. } \\
\text { var.'pride of automn' }\end{array}$ \\
\hline Polygonaceae & $\begin{array}{l}\text { Coccoloba uvifera } \mathrm{L} \text {. } \\
\text { Coccothrinax elegans } \mathrm{O} \text {. }\end{array}$ & Lamiaceae & $\begin{array}{l}\text { Coleus blumei Benth.var. } \\
\text { verschaffeltii Lem. }\end{array}$ \\
\hline Arecaceae & $\begin{array}{l}\text { Muñiz \& Borhidi 'Bella' } \\
\text { Coccothrinax fragrans Burret }\end{array}$ & $\begin{array}{l}\text { Lamiaceae } \\
\text { Borgoingceae }\end{array}$ & Coleus rehneltianus A. Berger \\
\hline Arecaceae & Cocos nucifera $\mathrm{L}$. & Boraginaceae & Cordia sebestana L. \\
\hline Euphorbiaceae & $\begin{array}{l}\text { Codiaeum frutescens } \\
\text { var.'Wellpark-Beauty' }\end{array}$ & Liliaceae & $\begin{array}{l}\text { Cordyline fruticosa (L.) } \\
\text { A.Chev. }\end{array}$ \\
\hline Euphorbiaceae & $\begin{array}{l}\text { Codiaeum gloriosum } \\
\text { superbum A.Juss }\end{array}$ & Liliaceae & $\begin{array}{l}\text { Cordyline terminalis (L.) } \\
\text { Kunth }\end{array}$ \\
\hline Euphorbiaceae & $\begin{array}{l}\text { Codiaeum varlegatum (L.) } \\
\text { A.Juss. } \\
\text { Codiaeum variegatum (L.) }\end{array}$ & Liliaceae & $\begin{array}{l}\text { Cordyline terminalis (L.) } \\
\text { Kunth var. amabilis }\end{array}$ \\
\hline Euphorbiaceae & $\begin{array}{l}\text { A.Juss. 'Clipper' } \\
\text { Codiaeum variegatum (L.) }\end{array}$ & Liliaceae & $\begin{array}{l}\text { Cordyline terminalis (L.) } \\
\text { Kunth var. calypso queen }\end{array}$ \\
\hline Euphorbiaceae & $\begin{array}{l}\text { A.Juss. var. 'delaruye' } \\
\text { Codiaeum variegatum (L.) }\end{array}$ & Liliaceae & $\begin{array}{l}\text { Cordyline terminalis (L.) } \\
\text { Kunth var. firebrand }\end{array}$ \\
\hline Euphorbiaceae & A.Juss. var.' america' & Liliaceae & $\begin{array}{l}\text { Cordyline terminalis (L.) } \\
\text { Kunth var. hawaii bonsai }\end{array}$ \\
\hline Euphorbiaceae & A.Juss. var. aucubifolium & Liliaceae & $\begin{array}{l}\text { Cordyline terminalis (L.) } \\
\text { Kunth var. in habitat }\end{array}$ \\
\hline Euphorbiaceae & $\begin{array}{l}\text { Codiaeum variegatum (L.) } \\
\text { A.Juss. var. cornutum }\end{array}$ & Liliaceae & $\begin{array}{l}\text { Cordyline terminalis (L.) } \\
\text { Kunth var. liliput }\end{array}$ \\
\hline Euphorbiaceae & $\begin{array}{l}\text { Codiaeum variegatum (L.) } \\
\text { A.Juss. var. 'elaine' }\end{array}$ & Arecaceae & Corypha umbraculifera $\mathrm{L}$. \\
\hline Euphorbiaceae & $\begin{array}{l}\text { Codiaeum variegatum (L.) } \\
\text { A.Juss. var. exotica }\end{array}$ & $\begin{array}{l}\text { Zingiberaceae } \\
\text { Zingiberaceae }\end{array}$ & $\begin{array}{l}\text { Costus afer Ker-Gawl. } \\
\text { Costus speciosus } \mathrm{Sm} .\end{array}$ \\
\hline Euphorbiaceae & $\begin{array}{l}\text { Codiaeum variegatum (L.) } \\
\text { A.Juss. var. imperialis }\end{array}$ & $\begin{array}{l}\text { Zingiberaceae } \\
\text { Bignoniaceae }\end{array}$ & $\begin{array}{l}\text { Costus spiralis } \text { Roscoe } \\
\text { Crescentia cujete } \mathrm{L} .\end{array}$ \\
\hline Euphorbiaceae & $\begin{array}{l}\text { Codiaeum variegatum (L.) } \\
\text { A.Juss. var. lineatum }\end{array}$ & Amaryllidaceae & Crinum asiaticum $\mathrm{L}$. \\
\hline Euphorbiaceae & $\begin{array}{l}\text { Codiaeum variegatum (L.) } \\
\text { A.Juss. var. punctum aureum }\end{array}$ & Amaryllidaceae & $\begin{array}{l}\text { Crinum procerum Carey ex } \\
\text { Herb. var. splendens Hannibal }\end{array}$ \\
\hline Euphorbiaceae & $\begin{array}{l}\text { Codiaeum variegatum (L.) } \\
\text { A.Juss. var.craigii }\end{array}$ & Acanthaceae & $\begin{array}{l}\text { Crossandra infundibuliformis } \\
\text { (L.) Nees. }\end{array}$ \\
\hline Euphorbiaceae & $\begin{array}{l}\text { Codiaeum variegatum (L.) } \\
\text { A.Juss. var.'General Paget' }\end{array}$ & $\begin{array}{l}\text { Acanthaceae } \\
\text { Euphorbiaceae }\end{array}$ & $\begin{array}{l}\text { Crossandra nilotica Oliv. } \\
\text { Croton zambesicus Müll. Arg. }\end{array}$ \\
\hline
\end{tabular}




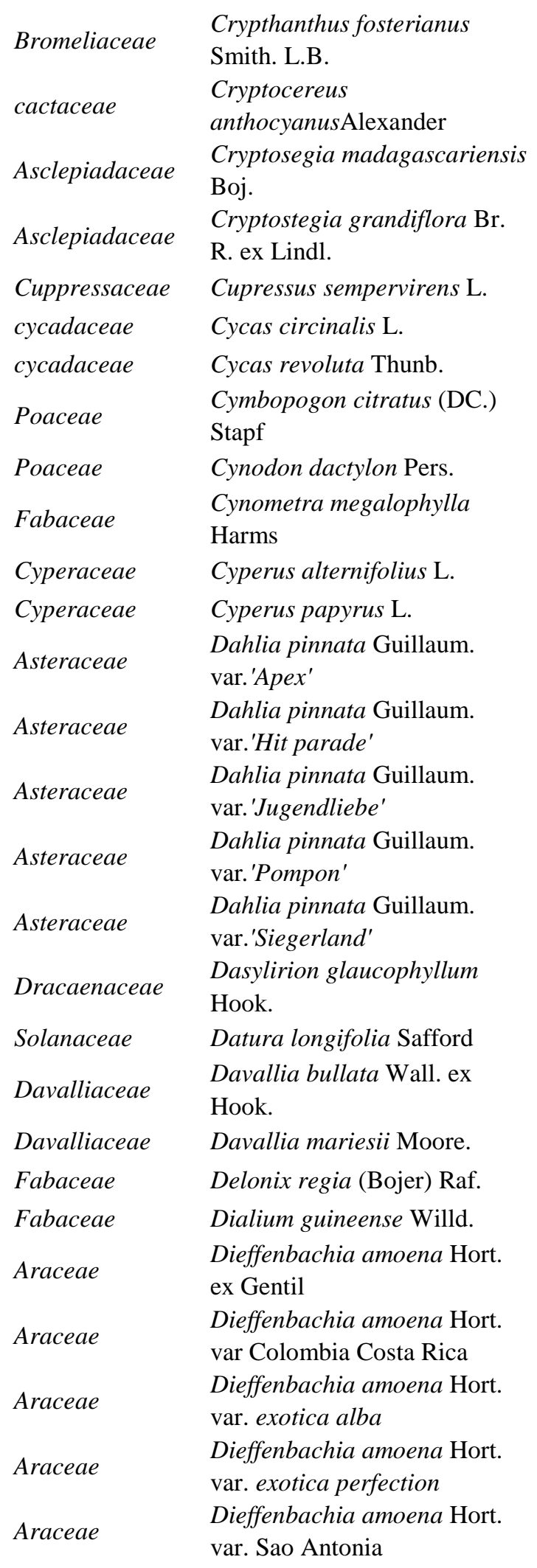

Araceae

Araceae

Araceae

araliaceae

araliaceae

Dracaenaceae

Dracaenaceae

Dracaenaceae

Dracaenaceae

Dracaenaceae

Dracaenaceae

Verbenaceae

Bromeliaceae

Crassulaceae

Crassulaceae

Cactaceae

Cactaceae

Cactaceae

Cactaceae

Pontederiaceae

Arecaceae

Zamiaceae

Zamiaceae

Gesneriaceae

Gesneriaceae

Gesneriaceae

Gesneriaceae
Dieffenbachia amoena Hort. var. Wilson's Delight Dieffenbachia maculata Schott. var. Rudolf Roehrs Dieffenbachia picta Schott. Dizygotheca elegantissima (Veitch ex Mast.) R. Vig. \& Guillaumin

Dizygotheca reginae (Linden ex W. Richards) Hemsl. Dracaena arborea (Willd.) Link

Dracaena godseffiana Sander ex Mast.

Dracaena godseffiana Sander ex Mast. var. friedmanii

Dracaena sanderiana Sander ex Mast.

Draceana fragans massangeana Ker-gawl.

Draceana marginata Ait.

Duranta repens L.

Dyckia fosteriana L.B.Sm.

Echeveria chihuahuaensis Poelln.

Echeveria elegans (Rose) A. Berger

Echinocactus grusonii Hildm. Echinocactus martini Cels ex K.Schum.

Echinocereus pectinatus Engelm.

Echinocereus triglochidiatus Eng. Eichhornia crassipes (Mart.) Solms

Elaeis guineensis Jacq. Encephalartos ferox G. Bertol. Encephalartos gratus Prain. Episcia cupreata (Hook.) Hanst. Episcia cupreata (Hook.) Hanst. var. 'frosty' Episcia cupreata (Hook.) Hanst. var.'acajou' Episcia cupreata (Hook.) Hanst. var.metallica 


\begin{tabular}{|c|c|c|c|}
\hline & Episcia cupreata (Hook.) & Onagraceae & Fuchsia speciosa Bailey \\
\hline eqe & Hanst.var.'Schimmer' & Agavaveae & Furcraea gigantea Vent. \\
\hline Gesneriaceae & $\begin{array}{l}\text { Episcia cupreata } \\
\text { (Hook.)Hanst. var. musaica }\end{array}$ & Onagraceae & Fuchsia triphylla L. \\
\hline Acanthaceae & $\begin{array}{l}\text { Eranthemum nervosum (VaL) } \\
\text { R. Br. } \\
\text { Erythrina indica Lamk. picta }\end{array}$ & Malpighiaceae & $\begin{array}{l}\text { Galphimia glauca Cav. } \\
\text { Gardenia jasminoides } \\
\text { fortuniana } \text { Ellis }\end{array}$ \\
\hline Fabaceae & & Asterace & Gerbera jamesonii Bolus ex \\
\hline Myrtaceae & Eucalyptus citriodora Hook. & & Adlam var. 'African daisies' \\
\hline Myrtaceae & Eucalyptus deglupta Blume & Asteraceae & $\begin{array}{l}\text { Gerbera jamesonii Bolus ex } \\
\text { Adlam var. rosea' }\end{array}$ \\
\hline Myrtaceae & $\begin{array}{l}\text { Eucalyptus torrelliana } \mathrm{F} \text {. } \\
\text { Muell. }\end{array}$ & Asteraceae & $\begin{array}{l}\text { Gerbera jamesonii Bolus ex } \\
\text { Adlam var.'Transvaal daisy }\end{array}$ \\
\hline Amaryllidaceae & $\begin{array}{l}\text { Eucharis grandiflora Planch. } \\
\text { \& Linden }\end{array}$ & Amaranthaceae & Gomphrena globosa L. \\
\hline Euphorbiaceae & Euphorbia lactea Haw. & Acanthaceae & Graptophyllum pictum Nees \\
\hline Euphorbiaceae & $\begin{array}{l}\text { Euphorbia millii Des Moul. } \\
\text { var. breonii }\end{array}$ & $\begin{array}{l}\text { Zygophyllaceae } \\
\text { Cactaceae }\end{array}$ & Guaiacum officinale $\mathrm{L}$. \\
\hline Euphorbiaceae & Euphorbia oncoclada Drake. & & (Cels) Br. \& R. \\
\hline Euphorbiaceae & Euphorbia tirucalli $\mathrm{L}$. & Asteraceae & Helianthus annuusL. \\
\hline Euphorbiaceae & Euphorbia $x$ keysii Hort. & Heliconiaceae & $\begin{array}{l}\text { Heliconia caribaea Lam. } \\
\text { 'Purpurea' }\end{array}$ \\
\hline Nymphaeaceae & Euryale ferox Salisb. & Heliconiaceae & Heliconia marginata Pittier \\
\hline Asteraceae & Felicia fruticosa Nichols. & Heliconiaceae & Heliconia psittacorum L. f. \\
\hline Cactaceae & $\begin{array}{l}\text { Ferocactus fordıl Britton \& } \\
\text { Rose }\end{array}$ & & Heliconia vellerigena Poepp. \\
\hline Cactaceae & $\begin{array}{l}\text { Ferocactus herresae } \\
\text { J.G.Ortega }\end{array}$ & $\begin{array}{l}\text { Urticaceae } \\
\text { Acanthaceae }\end{array}$ & $\begin{array}{l}\text { Helxine soleirolii } \text { Req. } \\
\text { Hemigraphis colorata Nees }\end{array}$ \\
\hline Cactaceae & Ferocactus latispinus Br. \& R. & Malvaceae & Hibiscus arnottianus A. Gray \\
\hline Cactaceae & $\begin{array}{l}\text { Ferocactus rectispinus } \\
\text { (Engelm.) L. D. Benson }\end{array}$ & Malvaceae & $\begin{array}{l}\text { Hibiscus rosa-sinensis L. } \\
\text { Hibiscus rosa-sinensis L. var. }\end{array}$ \\
\hline Moraceae & Ficus benjamina L. & Malvaceae & plenus \\
\hline Moraceae & $\begin{array}{l}\text { Ficus benjamina } \mathrm{L} . \\
\text { 'Variegata' }\end{array}$ & Malvaceae & $\begin{array}{l}\text { Hibiscus rosa-sinensis L. var. } \\
\text { albus }\end{array}$ \\
\hline $\begin{array}{l}\text { Moraceae } \\
\text { Moraceae }\end{array}$ & $\begin{array}{l}\text { Ficus benjamina } \text { L. 'Starlight' } \\
\text { Ficus elastica Roxb. }\end{array}$ & Malvaceae & $\begin{array}{l}\text { Hibiscus rosa-sinensis L. var. } \\
\text { california gold }\end{array}$ \\
\hline Moraceae & $\begin{array}{l}\text { Ficus elastica Roxb. } \\
\text { 'Doescheri' }\end{array}$ & Malvaceae & $\begin{array}{l}\text { Hibiscus rosa-sinensis L. var. } \\
\text { cooperi }\end{array}$ \\
\hline Moraceae & Ficus elastica Roxb.'Decora' & Malvaceae & $\begin{array}{l}\text { Hibiscus rosa-sinensis L. var. } \\
\text { crown of Bohemia }\end{array}$ \\
\hline Moraceae & $\begin{array}{l}\text { Ficus elastica } \\
\text { Roxb.'Schryveriana' }\end{array}$ & Malvaceae & $\begin{array}{l}\text { Hibiscus rosa-sinensis L. var. } \\
\text { mist }\end{array}$ \\
\hline Moraceae & Ficus pumila L. & & Hibiscus rosa-sinensis L. var. \\
\hline Moraceae & Ficus retusa $\mathrm{L}$. & Malvaceae & natal \\
\hline Moraceae & $\begin{array}{l}\text { Ficus rubiginosa Desf. ex } \\
\text { Vent. var. variegata Guilf. }\end{array}$ & Malvaceae & $\begin{array}{l}\text { Hibiscus rosa-sinensis L. var. } \\
\text { scarlet' }\end{array}$ \\
\hline Moraceae & $\begin{array}{l}\text { Ficus triangularis Warb. } \\
\text { Fittonia verschaffeltii } \mathrm{E} .\end{array}$ & Malvaceae & $\begin{array}{l}\text { Hibiscus schizopetalus (Mast.) } \\
\text { Hook. f. }\end{array}$ \\
\hline Aca & Coem. & Amaryllidaceae & Hippeastrum leopoldii Hort. \\
\hline
\end{tabular}


Amaryllidaceae

Amaryllidaceae

Araceae

Arecaceae

Asclepiadaceae

Euphorbiaceae

Asteraceae

Asteraceae

Rubiaceae

Rubiaceae

Rubiaceae

Rubiaceae

Rubiaceae

Rubiaceae

Rubiaceae

Rubiaceae

Bignoniaceae

Acanthaceae

Oleaceae

Oleaceae

Euphorbiaceae

Euphorbiaceae

Euphorbiaceae

Crassulaceae

Crassulaceae

Crassulaceae

Crassulaceae

Crassulaceae

Crassulaceae

Crassulaceae

Crassulaceae

Meliaceae
Hippeastrum puniceum (Lam.)

Kuntze

Hippeastrum reticulatum var. striatifolium (Herb.) Herb.

Homalomena wallisii Regel.

Howeia forsteriana Becc.

Huernia reticulata (Masson)

Haw.

Hura crepitans L.

Impatiens balsamina $\mathrm{L}$.

Impatiens marianae Rchb.f. ex

Hook.f.

Ixora chinensis Lamk.

Ixora coccinea $\mathrm{L}$.

Ixora finlaysoniana Wall. \&

G.Don

Ixora 'Herreras Pink'

Ixora javanica DC.

Ixora macrothyrsa Hort.

Ixora macrothyrsa Hort.

'Super King'

Ixora odorata Hook.

Jacaranda mimosaefolia Don D.

Jacobinia pohliana Benth. \&

Hook. f.

Jasminum nitidum Skan

Jasminum officinale L. affine

(Royle ex Lindl.) Rehder

Jatropha multifida L.

Jatropha pandurifolia Andr. Jatropha podagrica Hook.

Kaempferia pulchra Rodl.

Kalanchoe blossfeldiana

Poelln.

Kalanchoe blossfeldiana

Poelln.var.'Tom Thumb'

Kalanchoe daigremontiana

Hamet et Perr. de la Bat.

Kalanchoe fedtschenkoi

Hamet et Perr. de la Bat.

Kalanchoe gastonis-bonnieri Hamet et Perr. de la Bat.

Kalanchoe marmorata Bak.

Kalanchoe prolifera Bowie.

Khaya senegalensis (Desr.)
Lythraceae

Lamiaceae

Verbenaceae

Lythraceae

Arecaceae

Arecaceae

Arecaceae

Oleaceae

Arecaceae

Arecaceae

Arecaceae

Cactaceae

Cactaceae

Cactaceae

Cactaceae

Cactaceae

Cactaceae

Anacardiaceae

Marantaceae

Marantaceae

Marantaceae

Marantaceae

Meliaceae

Cactaceae

Cactaceae

Fabaceae

Bignoniaceae

Nyctaginaceae

Araceae

Araceae

Araceae
A.Juss.

Lagerstroemia indica L.

Lamium galeobdolon (L.) L. var. variegatum

Lantana camara L.

Lawsonia inermis L.

Licuala grandis $\mathrm{H}$. Wendl.

Licuala rumphii Blume

Licuala spinosa Wurmb.

Ligustrum ovalifolium Hassk.

Linospadix monostachya $\mathrm{H}$.

Wendl.

Livistona australis Mart.

Livistona chinensis Mart.

Malacocarpus tethracanthus

(Lem.) Rud.Mey.

Mammillaria bocasana

Poselg.

Mammillaria elegans DC.

Mammillaria macrothele Mart.

Mammillaria mainae

K.Brandegee

Mammillaria prolifera Haw.

Mangifera indica $\mathrm{L}$.

Maranta arundinacea L.

Maranta arundinacea $\mathrm{L}$. variegata

Maranta leuconeura E.

Morren.

Maranta leuconeura E.

Morren. erythroneura

G.S.Bunting

Melia azedarach $\mathrm{L}$.

Melocactus bahiensis (Br. \&

R.) Lutzelb.

Melocactus caesius H. L.

Weendl.

Millettia thonningii (Schum. \&

Thonn.) Baker

Millingtonia hortensis L. f.

Mirabilis jalapa L.

Monstera deliciosa Liebm.

Monstera epipremnoidesEngl.

Monstera friedrichsthalii

Schott 


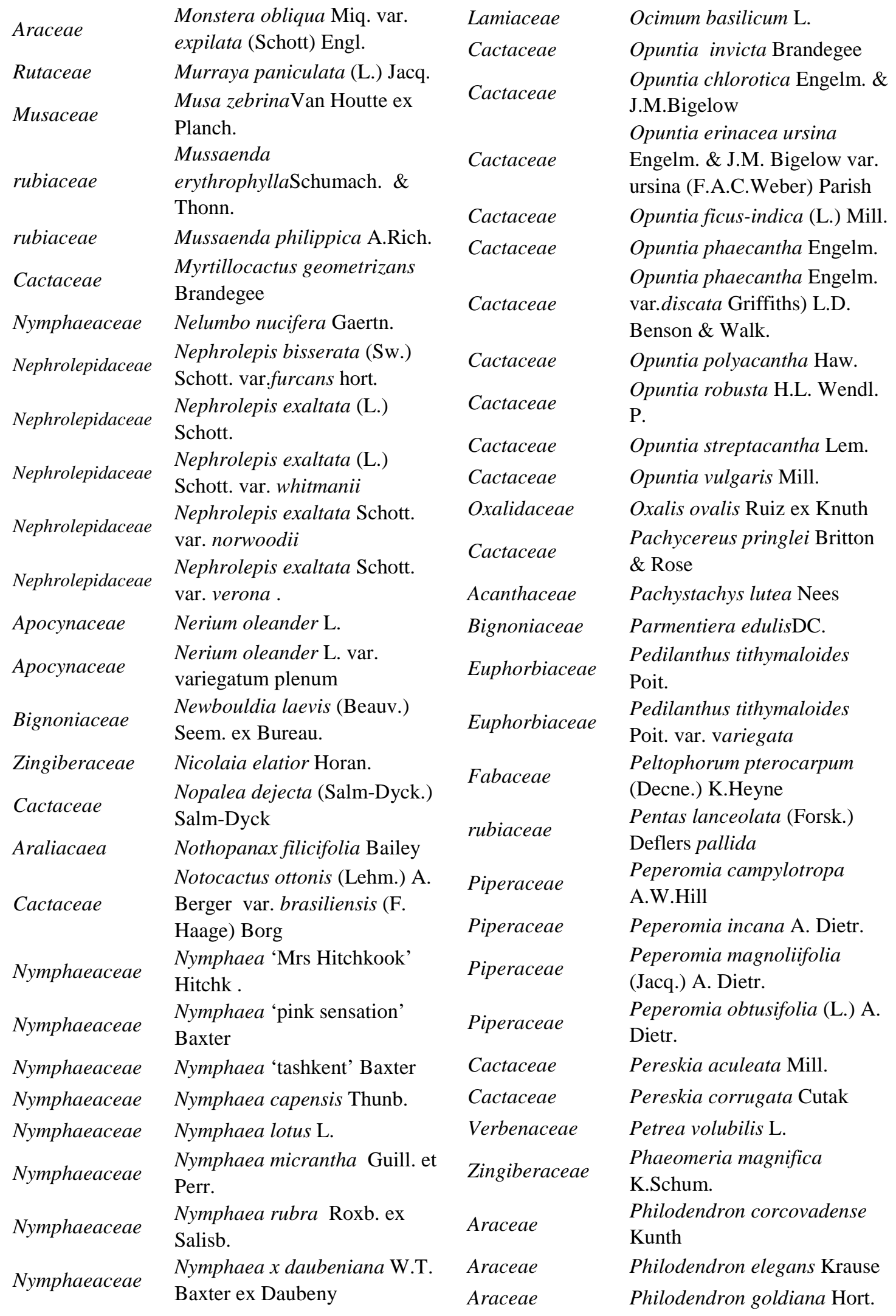




\begin{tabular}{|c|c|c|c|}
\hline Araceae & Philodendron 'Lynette' Hort. & Araliaceae & $\begin{array}{l}\text { Polyscias guilfoylei L.H. } \\
\text { Bailey laciniata }\end{array}$ \\
\hline Araceae & $\begin{array}{l}\text { Philodendron 'Majesty' Hort. } \\
\text { Philodendron scandens } \\
\text { K.Koch \& Sello }\end{array}$ & Araliaceae & $\begin{array}{l}\text { Polyscias guilgoylei L.H. } \\
\text { Bailey victorae Bailey }\end{array}$ \\
\hline Araceae & $\begin{array}{l}\text { Philodendron williamsii Hook. } \\
\text { f. } \\
\text { Philodendron } x \text { corsinianum }\end{array}$ & Araliaceae & $\begin{array}{l}\text { Polyscias palapala Hort. } \\
\text { Polyscias scutellaria (Burm.f.) } \\
\text { Fosberg }\end{array}$ \\
\hline Araceae & Hort. & Portulacaceae & Portulaca grandiflora Hook. \\
\hline Araceae & $\begin{array}{l}\text { Philodendron x Florida } \\
\text { compacta Hort. }\end{array}$ & Portulacaceae & $\begin{array}{l}\text { Portulaca grandiflora Hook. } \\
\text { florepleno }\end{array}$ \\
\hline Euphorbiaceae & Phyllanthus angustifolius Sw. & Arecaceae & Pritchardia beccariana Rock \\
\hline Pinaceae & Pinus canariensis $\mathrm{Sm}$. & Acanthaceae & Pseuderanthemum \\
\hline Pinaceae & Pinus caribaea Morelet & Acantracease & atropurpureum var.tonga \\
\hline Fabaceae & $\begin{array}{l}\text { Pithecellobium dulce(Roxb.) } \\
\text { Benth. }\end{array}$ & Acanthaceae & $\begin{array}{l}\text { Pseuderanthemum reticulatum } \\
\text { Radlk. }\end{array}$ \\
\hline Polypodiaceae & $\begin{array}{l}\text { Platycerium angolense Welm. } \\
\text { ex Hook. }\end{array}$ & $\begin{array}{l}\text { Pteridaceae } \\
\text { Pteridaceae }\end{array}$ & $\begin{array}{l}\text { Pteris cretica L. } \\
\text { Pteris ensiformis Burm. }\end{array}$ \\
\hline Polypodiaceae & $\begin{array}{l}\text { Platycerium bifurcatum (Cav.) } \\
\text { C.Chr. }\end{array}$ & $\begin{array}{l}\text { Punicaceae } \\
\text { Punicaceae }\end{array}$ & $\begin{array}{l}\text { Punica granatum } \mathrm{L} . \\
\text { Punica granatum L. florepleno }\end{array}$ \\
\hline Polypodiaceae & Platycerium hilii Moore & Bromeliaceae & Puya caerulea $\mathrm{L}$. \\
\hline Polypodiaceae & $\begin{array}{l}\text { Platycerium stemaria (P. } \\
\text { Beauv.) Desv. }\end{array}$ & Combretaceae & Quisqualis indica $\mathrm{L}$. \\
\hline Polypodiaceae & $\begin{array}{l}\text { Platycerium superbum } \\
\text { Joncheere \& Hennipman }\end{array}$ & Strelitziaceae & $\begin{array}{l}\text { Ravenala madagascariensis } \\
\text { Gmel. J.F. }\end{array}$ \\
\hline Lamiaceae & $\begin{array}{l}\text { Plectranthus coleoides } \\
\text { Benth.var. marginatus }\end{array}$ & $\begin{array}{l}\text { Arecaceae } \\
\text { Cactaceae }\end{array}$ & $\begin{array}{l}\text { Rhapis excelsa Henry } \\
\text { Rhipsalis cassutha Gaertn. }\end{array}$ \\
\hline & $\begin{array}{l}\text { Plectranthus fruticosus Wight } \\
\text { ex Hook.f. }\end{array}$ & Commelinaceae & $\begin{array}{l}\text { Rhoeo discolor (L'Hér.) Hance } \\
\text { Rhoeo spathacea (Sw.) Stearn }\end{array}$ \\
\hline Plumbaginaceae & Plumbago capensis Thunb. & nelinaceae & vittata \\
\hline Apocynaceae & Plumeria alba $\mathrm{L}$. & Cactaceae & Richycereus weberi Hort. \\
\hline Apocynaceae & $\begin{array}{l}\text { Plumeria rubraf. tricolor } \\
\text { (Roem. \& Pav.) Woodson }\end{array}$ & Rosaceae & $\begin{array}{l}\text { Rosa centifolia } \mathrm{L} . \\
\text { Rosa floribunda } \text { Hort. ex }\end{array}$ \\
\hline Apocynaceae & Plumeria rubra $\mathrm{L}$. & Rosaceae & Andrews 'Crimson Rosette' \\
\hline Agavaceae & $\begin{array}{l}\text { Polianthes tuberosa L. } \\
\text { Polyalthia longifolia (Sonn.) }\end{array}$ & Rosaceae & $\begin{array}{l}\text { Rosa floribunda Hort. ex } \\
\text { Andrews 'Fashionette' }\end{array}$ \\
\hline Annonaceae & $\begin{array}{l}\text { Hook. f. \& Thomson } \\
\text { Polyalthia longifolia (Sonn.) }\end{array}$ & Rosaceae & $\begin{array}{l}\text { Rosa floribunda Hort. ex } \\
\text { Andrews 'Scarlet Marvel' }\end{array}$ \\
\hline Annonaceae & $\begin{array}{l}\text { Hook. f. \& Thomson var. } \\
\text { pendula }\end{array}$ & Rosaceae & $\begin{array}{l}\text { Rosa floribunda Hort. ex } \\
\text { Andrews 'Spice' }\end{array}$ \\
\hline $\begin{array}{l}\text { Polypodiaceae } \\
\text { Polypodiaceae }\end{array}$ & $\begin{array}{l}\text { Polypodium musifolium } \mathrm{B} 1 . \\
\text { Polypodium ponctatum Thunb. }\end{array}$ & Rosaceae & $\begin{array}{l}\text { Rosa floribunda Hort. ex } \\
\text { Andrews 'Double Paul's } \\
\text { Scarlet' }\end{array}$ \\
\hline Araliaceae & $\begin{array}{l}\text { Polyscias balfouriana Bailey } \\
\text { Polyscias balfouriana Bailey }\end{array}$ & Rosaceae & $\begin{array}{l}\text { Rosa grandiflora Lindl. 'Queen } \\
\text { Elisabeth' }\end{array}$ \\
\hline Araliaceae & $\begin{array}{l}\text { pennockii } \\
\text { Polyscias filicifolia Bailey }\end{array}$ & Rosaceae & $\begin{array}{l}\text { Rosa odorata Sw. 'Better } \\
\text { Times' }\end{array}$ \\
\hline Araliaceae & $\begin{array}{l}\text { Polyscias guilfoylei L.H. } \\
\text { Bailey }\end{array}$ & Rosaceae & $\begin{array}{l}\text { Rosa odorata Sw. 'Golden } \\
\text { Rapture' }\end{array}$ \\
\hline
\end{tabular}




\begin{tabular}{|c|c|c|c|}
\hline Rosaceae & Rosa odorata Sw. 'Happiness' & Verbenaceae & Stachytarpheta cayenensis \\
\hline Rosaceae & $\begin{array}{l}\text { Rosa odorata Sw. 'Mrs. Pierre } \\
\text { S. Dupont' }\end{array}$ & Apocynaceae & $\begin{array}{l}\text { (L.C. Rich.) Schau. } \\
\text { Stapelia asterias Masson }\end{array}$ \\
\hline Rosaceae & $\begin{array}{l}\text { Rosa odorata Sw. 'Mrs. W.C. } \\
\text { Miller' }\end{array}$ & Apocynaceae & $\begin{array}{l}\text { Stapelia gigantea N.E. Br. } \\
\text { Stapelia gigantea N.E. Br. }\end{array}$ \\
\hline Arecaceae & Roystonea regia O.F. Hook. & Аросупасеае & var.palllida E. Phillips \\
\hline Acanthaceae & $\begin{array}{l}\text { Ruellia squarrosa (Fenzl) } \\
\text { Cufod. }\end{array}$ & Apocynaceae & Stapelia nobilisN.E.Br. \\
\hline Fabaceae & $\begin{array}{l}\text { Samanea saman (Jacquin) } \\
\text { Merrill }\end{array}$ & Strelitziaceae & $\begin{array}{l}\text { Stenotaphrum secundatum } \\
\text { (Walt.) Kuntze. Variegatum } \\
\text { Strelitzia reginae Pittier }\end{array}$ \\
\hline Acanthaceae & Sanchezia nobilis Hook.f. & Acanthaceae & Strobilanthes dyerianus Mast. \\
\hline Dracaenaceae & Sansevieria cylindrica Boj. & Acanthaceae & Strobilanthes maculata Nees. \\
\hline Dracaenaceae & $\begin{array}{l}\text { Sansevieria stuckyi Godegroy- } \\
\text { Leboeuf. }\end{array}$ & Apocynaceae & $\begin{array}{l}\text { Strophanthus gratus (Hook.) } \\
\text { Franch. }\end{array}$ \\
\hline Dracaenaceae & $\begin{array}{l}\text { Sansevieria trifasciata } \text { Prain. } \\
\text { Sansevieria trifasciata } \text { Prain. }\end{array}$ & Araceae & $\begin{array}{l}\text { Syngonium macrophyllum } \\
\text { Engl. }\end{array}$ \\
\hline Dracaenaceae & $\begin{array}{l}\text { var. hahnii } \\
\text { Sansevieria trifasciata Prain. } \\
\text { var. hahnii variegata }\end{array}$ & Araceae & $\begin{array}{l}\text { Syngonium podophyllum } \\
\text { Schott. albo-virens }\end{array}$ \\
\hline Dracaenaceae & $\begin{array}{l}\text { var. hahnii variegata } \\
\text { Sansevieria trifasciata } \\
\text { Prain.var. golden hahnii }\end{array}$ & Araceae & $\begin{array}{l}\text { Syngonium podophyllum } \\
\text { Schott. 'emerald gem' }\end{array}$ \\
\hline Dracaenaceae & $\begin{array}{l}\text { Prain.var. golden hannıl } \\
\text { Sansevieria trifasciata }\end{array}$ & Araceae & $\begin{array}{l}\text { Syngonium podophyllum } \\
\text { Schott. 'green gold' }\end{array}$ \\
\hline & Prain.var. laurentii' & Asteraceae & Tagetes patula $\mathrm{L}$. \\
\hline Araliaceae & $\begin{array}{l}\text { schefflera arborlcola (Hayata) } \\
\text { Merr. }\end{array}$ & Bignoniaceae & $\begin{array}{l}\text { Tecoma stans (L.) Juss. ex } \\
\text { Kunth. }\end{array}$ \\
\hline Araliaceae & $\begin{array}{l}\text { Schefflera farinosa (Blume) } \\
\text { Merr. }\end{array}$ & Lamiaceae & Tectona grandisL. f. \\
\hline Araceae & $\begin{array}{l}\text { Schismatoglottis novo- } \\
\text { guineensis Br. N.E. }\end{array}$ & $\begin{array}{l}\text { Combretaceae } \\
\text { Combretaceae }\end{array}$ & $\begin{array}{l}\text { Terminalia cattapa L. } \\
\text { Terminalia mantaly H.Perrier }\end{array}$ \\
\hline Araceae & $\begin{array}{l}\text { Schizocasia lauterbachiana } \\
\text { Engl. }\end{array}$ & Malvaceae & $\begin{array}{l}\text { Thespesia populnea Soland. ex } \\
\text { Corrêa }\end{array}$ \\
\hline $\begin{array}{l}\text { Araceae } \\
\text { Cyperaceae }\end{array}$ & $\begin{array}{l}\text { Scindapsus aureus Engl. } \\
\text { Scirpus cernuus Vahl. }\end{array}$ & Apocynaceae & $\begin{array}{l}\text { Thevetia neriifolia Juss. ex } \\
\text { Steud. }\end{array}$ \\
\hline & $\begin{array}{l}\text { Setcreasea purpurea B.M. } \\
\text { Boom }\end{array}$ & Apocynaceae & $\begin{array}{l}\text { Thevetia peruviana (Pers.) } \\
\text { Merr. }\end{array}$ \\
\hline Araceae & $\begin{array}{l}\text { Spathiphyllum blandum } \\
\text { Schott. }\end{array}$ & $\begin{array}{l}\text { Malpighiaceae } \\
\text { Cupressaceae }\end{array}$ & $\begin{array}{l}\text { Thryallis glauca O. Ktze } \\
\text { Thuja occidentalis L. }\end{array}$ \\
\hline Araceae & $\begin{array}{l}\text { Spathiphyllum cannaefolium } \\
\text { Schott. }\end{array}$ & Acanthaceae & $\begin{array}{l}\text { Thunbergia alata Boj. } \\
\text { Thunbergia erecta (Benth.) T. }\end{array}$ \\
\hline Araceae & Spathiphyllum 'Mc Coy' & $e$ & Anders. \\
\hline Bignoniaceae & $\begin{array}{l}\text { Spathodea campanulata } \mathrm{P} . \\
\text { Beauv. }\end{array}$ & Acanthaceae & $\begin{array}{l}\text { Thunbergia fragrans Roxb. } \\
\text { Thunbergia grandiflora }\end{array}$ \\
\hline Orchidaceae & Spathoglottis plicata Blume & $e$ & (Roxb. ex Rottl.) Roxb. \\
\hline Asteraceae & $\begin{array}{l}\text { Spilanthes fusca Hort. Par. ex } \\
\text { Lam. }\end{array}$ & Asteraceae & Tithonia diversifolia A.Gray \\
\hline Asteraceae & Spilanthes oleracea L. & Commelinace & variegata \\
\hline
\end{tabular}


Commelinaceae

Zygophyllaceae

Liliaceae

Turneraceae

Orchidaceae

Orchidaceae

Orchidaceae

Nymphaeaceae

Nymphaeaceae

Nymphaeaceae

Arecaceae

Asteraceae

Araceae
Tradescantia fluminensis Vell. Quicksilver

Tribulus cistoides L.

Tulipa gesnerana L. var.

Blizzard

Turnera ulmifolia L.

Vanda 'Miss Agnes Joachim'

Vanda caerulea Griff. ex

Lindl.

Vanda teres Lindl.

Victoria amazonica Sowerby

Victoria regia Lindl.

Victoria regia Lindl. var. cruziana Orbign.

Washingtonia filifera Wendl.

Wedelia trilobata (L.) Hitch.

Xanthosoma lindenii (André)

S.Moore magnificum
Araceae

Agavaceae

Agavaceae

Zamiaceae

Zamiaceae

Zamiaceae

Zamiaceae

Zamiaceae

Zamiaceae

Zamiaceae

Zamiaceae

Commelinaceae

Amaryllidaceae

Asteraceae

Poaceae
Xanthosoma lindenii (André)

S.Moore 'yautia'

Yucca aloifolia L.

Yucca baccata Torr.

Zamia dominican L.f.

Zamia dudgesiana L.f.

Zamia fischeri Miq.

Zamia floridana A. DC.

Zamia furfuracea L.f.

Zamia fusca Hort.Paull. ex

Regel

Zamia pumila L.

Zamia standleyi Schutzman

Zebrina pendula Schnizl.

Zephyranthes carinata Herb.

Zinnia elegans Jacq.

Zoysia tenuifolia Trin.

Tableau 1 : Diversité taxonomique de la flore horticole dans différentes villes du Togo.

\begin{tabular}{|c|c|c|c|c|c|c|c|c|c|}
\hline \multirow{3}{*}{$\begin{array}{l}\text { Groupes } \\
\text { taxonomiques }\end{array}$} & \multicolumn{9}{|c|}{ Nombre de taxons } \\
\hline & \multicolumn{3}{|c|}{ Lomé } & \multicolumn{3}{|c|}{ Atakpamé } & \multicolumn{3}{|c|}{ Kpalimé } \\
\hline & Familles & Genres & Espèces & Familles & Genres & Espèces & Familles & Genres & Espèces \\
\hline Ptéridophytes & 4 & 6 & 20 & 0 & 0 & 0 & 2 & 2 & 3 \\
\hline Gymnospermes & 4 & 8 & 17 & 1 & 1 & 1 & 4 & 5 & 8 \\
\hline Dicotylédones & 46 & 145 & 315 & 31 & 70 & 92 & 37 & 76 & 98 \\
\hline Monocotylédones & 18 & 87 & 260 & 10 & 22 & 30 & 11 & 20 & 29 \\
\hline Total & 72 & 246 & 612 & 42 & 93 & 123 & 54 & 103 & 138 \\
\hline
\end{tabular}

L'indice de Shannon atteint la valeur de 5,98. L'équitabilité de Piélou atteint la valeur 0,78. L'indice d'hétérogénéité de Simpson est de 0,96 . Les six familles les plus riches en nombre de genres, renferment plus de $34 \%$ des effectifs. Ce sont les Arecaceae (18 genres), Cactaceae (17), Acanthaceae (15), Araceae (14), Asteraceae (10), Liliaceae (10). Elles sont suivies dans l'ordre décroissant par les Euphorbiaceae (8), les Apocynaceae et Bignoniaceae (7 genres chacune), les Caesalpiniaceae, Verbenaceae, Bromeliaceae, Poaceae avec 6 genres chacune, les Asclepiadaceae, Araliaceae et Zingiberaceae (5 genres chacune). Les dix familles les mieux représentées comptent pour plus de $45 \%$ des effectifs tandis que les dix familles les moins représentées (1 espèce chacune) rassemblent moins de $2 \%$ des effectifs (Figure 1).

Les genres les plus diversifiés sont dans l'ordre décroissant Begonia (12 espèces), Opuntia (10), Philodendron (9 espèces chacun) Ixora et Nymphaea (8 espèces), Calathea, Kalanchoe et Zamia (7 espèces chacun). Ils sont suivis des genres Anthurium et Ficus avec chacun 6 espèces. Les genres à 5 espèces sont: Agave, Alocasia, Aloe, Citrus, Clerodendron, Dracaena, Euphorbia, Mammillaria, Platycerium et Spathiphyllum. 
Les genres ayant moins de 5 espèces sont au nombre de 238 (Figure 2) et regroupent à eux seuls 397 espèces, soit environ $83 \%$ des espèces horticoles recensées.

Parmi les espèces à plusieurs variétés, on retient Codiaeum variegatum (14), Hibiscus rosa-sinensis (9) Canna generalis (7). Les espèces suivantes ont chacune 6 variétés: Acalypha wilkesiana, Caladium hortulanum, Cordyline terminalis, Dieffenbachia amoena, Episcia cupreata, Nephrolepis exaltata et Rosa floribunda. Les espèces à 5 variétés sont: Coleus blumei, Dahlia pinnata, Polyscias balfouriana, Rosa odorata et Sansevieria trifasciata.

Les espèces de plus de 6 variétés regroupent $12 \%$ des plantes recensées alors que les autres, au nombre de 234 regroupent 296 variétés, soit environ $78 \%$ des plantes horticoles du Togo (Figure 2). Les espèces les mieux représentées (indice de raréfaction RI inférieur à 80\%) sont au nombre de 274 et représentent $44,77 \%$ des espèces inventoriées. Les 15 espèces les plus fréquentes sont par

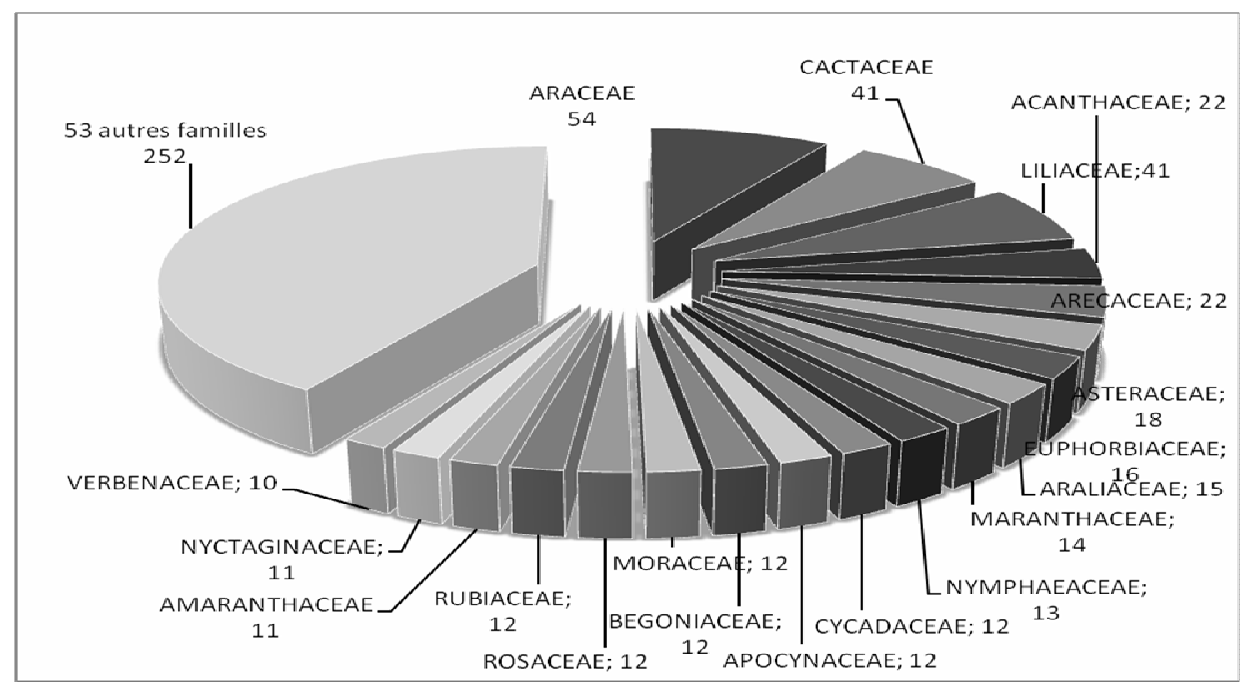

Figure 1 : Spectre des familles (les chiffres indiquent le nombre d'espèces).

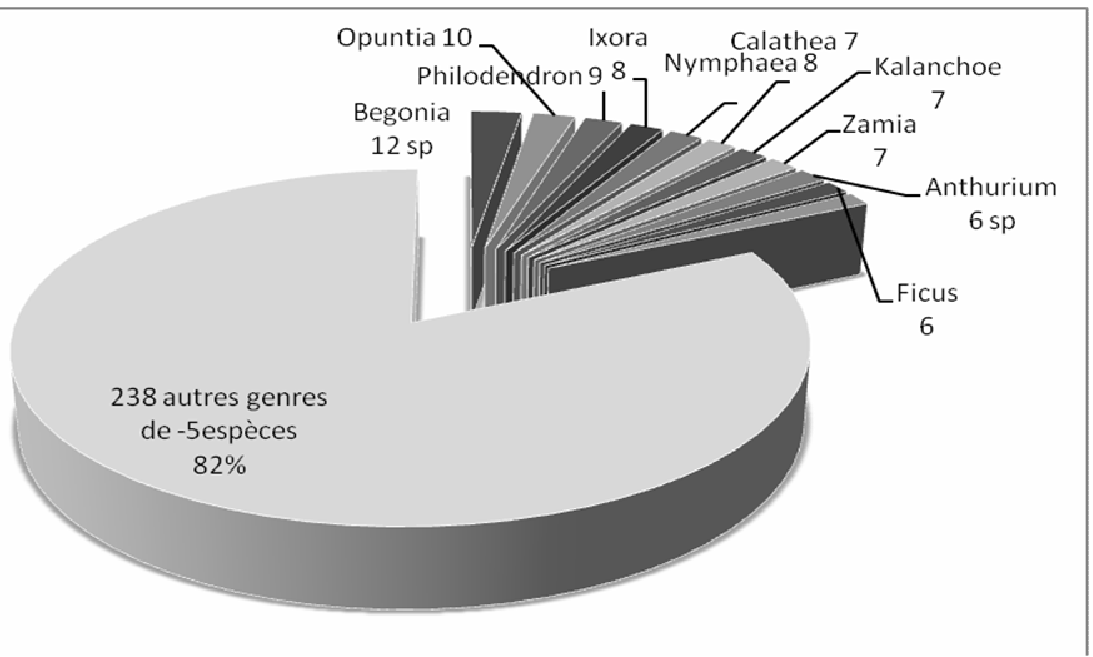

Figure 2 : Spectre des genres (les chiffres indiquent le nombre d'espèces). 
ordre alphabétique Aloe vera var. chinensis, Bougainvillea glabra, Catharanthus roseus, Cordyline terminalis, Dieffenbachia amoena, Dracaena arborea, Erythrina indica var. picta, Euphorbia millii var. breonii, Ficus bengamina, Ficus retusa, Ixora macrothyrsa, Murraya paniculata, Nerium oleander, Pithecellobium dulce, et Polyalthia longifolia.

Les espèces rares (indice de raréfaction supérieur ou égal à $80 \%$ ) sont au nombre de 338 (soit 55,23\% du total). Ces espèces se retrouvent dans moins de $10 \%$ des exploitations et stations horticoles. Parmi elles, 67 ne sont présentes que dans une seule pépinière (RI $\geq 98,18 \%$ ). On peut citer Calathea makoyana (Maranthaceae); Cryptostegia grandiflora (Asclepiadaceae); Davalia bullata (Davalliaceae); Echinocereus pectinatus (Cactaceae); Ficus elastica var. decora (Moraceae); Peperomia clusiifolia (Peperomiaceae); Monstera deliciosa (Araceae) ; Tithonia diversifolia (Asteraceae); Vanda caerulea (Orchidaceae); Zamia furfuracea (Zamiaceae).

Les différents types morphologiques composant la flore horticole du Togo sont présentés à la Figure 3. Ce spectre indique que les herbes $(\mathrm{H})$ sont les plus abondantes avec $55,23 \%$ suivies des arbustes (As) et les arbres (A) avec respectivement $16 \%$ et $15 \%$ des espèces. Les lianes (L), les plantes aquatiques (Hq) et les sous-arbrisseaux (sAs) constituent les types morphologiques les moins représentés. Les plantes ligneuses sont moyennement représentées avec 173 espèces pour un taux spécifique de 28 (Figure 3 ).

Les espèces horticoles sont originaires des cinq continents. Plus de la moitié (52\%) proviennent des Amériques contre 18\% pour l'Afrique. En dehors des Amériques (30\%) et de l'Asie (25\%), chacun des 3 autres continents renferment $15 \%$ des Ptéridophytes inventoriés. Les Gymnospermes sont à 53\% originaires des Amériques et seulement 10\% provient $\mathrm{du}$ continent africain. Pour les Monocotylédones, les espèces originaires d'Afrique ne représentent que $15 \%$ de l'ensemble. Ce pourcentage est de $20 \%$ pour les Dicotylédones. Parmi les Dicotylédones, les plantes originaires des Amériques rassemblent à elles seules 54\% du total (Tableau 3).

Chez les plantes recensées, différents organes sont à l'origine de leur esthétique. On distingue :

- 311 espèces regroupées en 106 genres utilisées pour leur feuillage décoratif dont Alocasia plumbea, Alocasia sanderiana, Dieffenbachia amoena (Araceae) et de Alpinia sanderae (Zingiberaceae);

- 69 espèces appartenant à 12 familles sont utilisées à cause de leur allure ou port décoratif. C'est le cas de Ravenala madagascariensis (Strelitziaceae), Polyalthia longifolia (Annonaceae), Araucaria cunninghamii, Callitris intratropica (Cuppressaceae) et de Cycas revoluta (Cycadaceae) ;

- 166 espèces réparties dans 37 familles sont utilisées pour leur floraison. Il s'agit entre autres de Allamanda cathartica (Apocynaceae); Gardenia jasminoides (Rubiaceae); Guaiacum officinale (Zygophyllaceae); Hibiscus rosa-sinensis (Malvaceae); Mussaenda philippica (Rubiaceae); Plumbago capensis (Plumbaginaceae) :

- 9 espèces sont cultivées pour le parfum de leurs feuilles, fleurs ou fruits. C'est le cas de Cymbopogon citratus (Poaceae), Polyanthes tuberosa (Liliaceae), Cynometra megalophylla (Caesalpiniaceae) et de Murraya paniculata (Rutaceae).

Mais en fonction des saisons, ces caractéristiques (feuillage, port/allure, floraison, fruits) peuvent se combiner pour donner un aspect ornemental ou décoratif plus attrayant.

\section{DISCUSSION}

Les exploitations de plantes ornementales sont généralement de petites superficies $\left(288 \pm 202 \mathrm{~m}^{2}\right)$ installées en bordure des routes. Cette étroitesse des exploitations et leur emplacement n'est pas un caractère spécifique au Togo, mais s'observe également dans d'autres pays de la sous région (Cissé, 1998 ; Brock et Foeken, 2006).

La richesse floristique des pépinières ou exploitations horticoles est très élevée; 


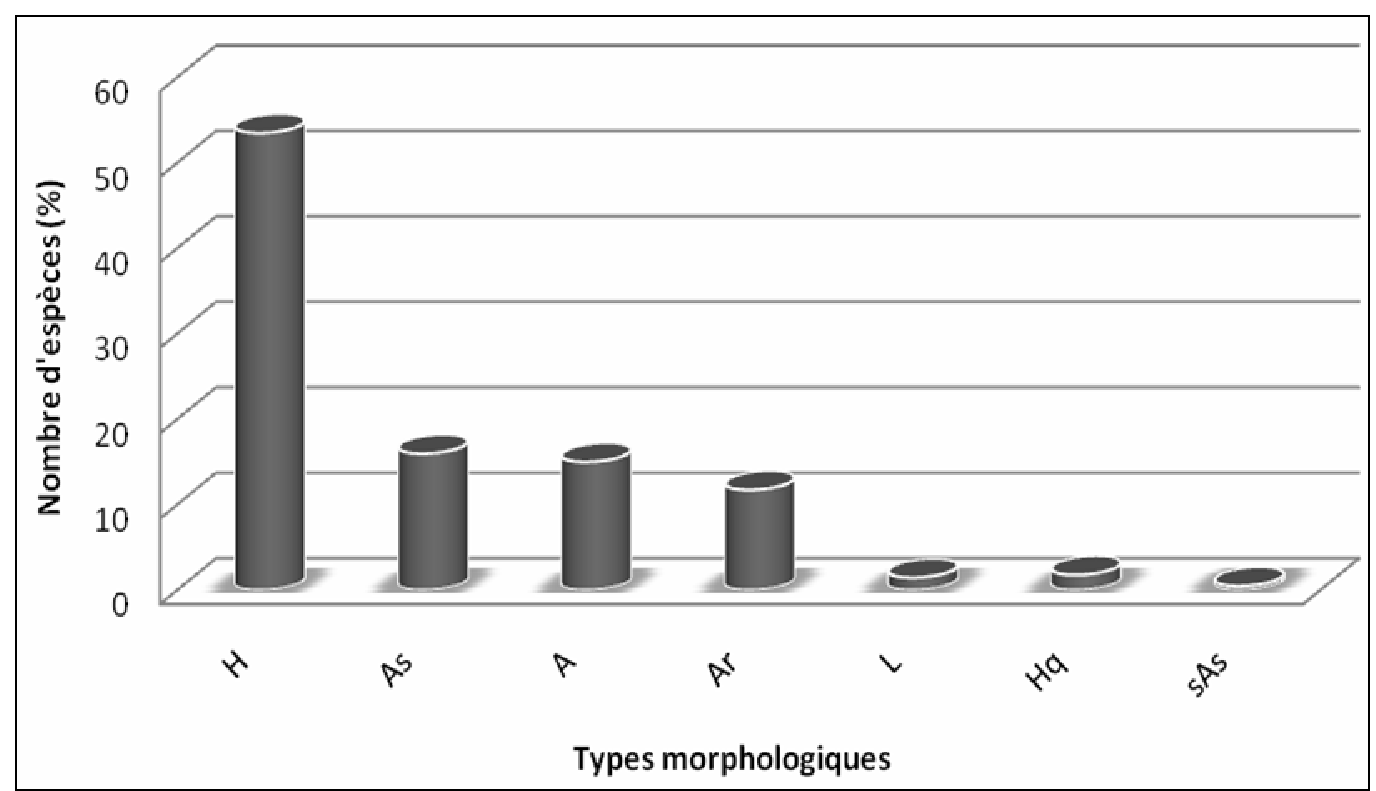

(A : arbre, Ar: arbuste, As : arbrisseau, $H:$ herbe, $H q:$ herbe aquatique, L : liane, sAs : sous-arbrisseau)

Figure 3: Types morphologiques de la flore horticole du Togo.

Tableau 2 : Répartition des espèces suivant les continents et les groupes taxonomiques.

\section{Continents}

\section{Groupes Taxonomiques}

\begin{tabular}{cccc}
\hline Ptéridophytes & Gymnospermes & Dicotylédones & Monocotylédones \\
\hline 3 & 2 & 67 & 35 \\
6 & 10 & 185 & 120 \\
5 & 3 & 66 & 45 \\
3 & 1 & 12 & 18 \\
3 & 3 & 12 & 13 \\
$\mathbf{2 0}$ & $\mathbf{1 9}$ & $\mathbf{3 4 2}$ & $\mathbf{2 3 1}$ \\
$\mathbf{3 , 2 7 \%}$ & $\mathbf{3 , 1 0 \%}$ & $\mathbf{5 5 , 8 8 \%}$ & $\mathbf{3 7 , 7 5 \%}$ \\
\hline
\end{tabular}

612 espèces, soit $18 \%$ de la flore togolaise. Ce chiffre est en hausse par rapport à celui de Radji (1998) qui a recensé 426 espèces. Cette différence de richesse laisse penser que de nouvelles espèces auraient été introduites puisque ce sont les mêmes exploitations qui avaient fait l'objet d'étude auparavant. Un inventaire périodique est donc nécessaire pour suivre l'évolution de l'horticulture ornementale au Togo.

La flore horticole est regroupée en 72 familles dont les mieux représentées, c'est-àdire ayant plus de visibilité dans le pays, sont les Nyctaginaceae avec le genre 
Bougainvillea, les Annonaceae avec le genre Polyalthia et les Rubiaceae avec le genre Murraya. Ces genres sont caractérisés par un mode de reproduction aisé (bouturage et semence) et une forte demande. Ce qui explique leur importante fréquence dans le paysage. L'ordre d'importance des familles botaniques recensées est très proche de celui observé en Côte d'Ivoire, notamment dans les villes d'Abidjan et de San Pédro (Aké Assi, 2002). Mais le nombre moyen d'espèces recensées (246) par exploitation dans cette étude est assez élevé par rapport aux recensements réalisés en Côte d'Ivoire (Aké Assi, 2002).

L'horticulture ornementale au Togo, à l'instar de celle de la plupart des pays africains, est malheureusement trop orientée vers des taxons exotiques; seulement $18 \%$ des taxons recensés sont originaires du continent africain et environ 40 espèces $(6,53 \%)$ sont signalées dans la flore du Togo (Brunel et al., 1984). Les espèces africaines recensées présentent des critères très recherchés par les exploitants et la clientèle. C'est le cas de Quisqualis indica (Combretaceae), Baissea multiflora (Apocynaceae) ou de Gardenia erubescens (Rubiaceae). Cet état de fait pose le problème de la valorisation des espèces des flores autochtones d'Afrique.

\section{Conclusion}

Cette étude a permis de faire l'état des lieux sur les connaissances actuelles de la flore horticole togolaise. La répartition de ces espèces dans les grands groupes taxonomiques, indique que les monocotylédones, sont majoritairement représentés avec en tête les Araceae, suivie des Liliaceae. En considérant tous les types biologiques, les herbacées sont les mieux représentées dans tous les relevés: 328 espèces soit $53,51 \%$ du total. La flore locale très faiblement représentée $(6,53 \%)$, mérite d'être mieux valorisée, surtout qu'elle est très sollicitée. Il convient de mieux explorer cette flore locale et envisager des techniques de multiplication modernes (notamment la culture in vitro) pour promouvoir leur usage comme plante ornementale.

\section{BIBLIOGRAPHIE}

Aké Assi AE. 2002. Contribution à l'étude des plantes ornementales cultivées dans les régions d'Abidjan et de San-Pedro, en Côte d'Ivoire. Volume 1 - Textes et iconographies. Th. Doct. 3e cycle, Univ. Cocody (RCI), p. 197.

Akpagana K. 1992. Espèces nouvelles pour la flore du Togo. Ann. Univ. Bénin, Sect. Sci., 10: 25-32.

Berhaut J. 1971-1988. Flore Illustrée du Sénégal, Dicotylédones. Tomes I, II, IV, V, VI, IX. Presses de l'Imprimerie Maisonnneuve : Sainte-Rufine, France.

Brock B, Foeken D. 2006. Urban horticulture for a better environment: A case study of Cotonou, Benin. Habitat International, 30 : 558-578.

Brunel JF, Hiepko P, Scholz H, 1984. Flore Analytique du Togo. GTZ édn: Eschborn; 750.

Byrd Graf A. 1981. Tropica: Color Cyclopedia of Exotic Plants and Trees $\left(2^{\mathrm{e}}\right.$ édn). Roehrs Company East Rutherford: N.J., USA; 1136.

Cisse O. 1998. Horticulture urbaine et l'environnement. Paper presented at the IDRC workshop on "The contribution of urban agriculture to food security in West African Cities", Ouagadougou, 15-18 June, 1998.

Fleury A, Ba A, To HTT. 2008. Renouvellement du concept d'agriculture urbaine dans les villes du sud. Revue Open House International: : 5-13.

Fongnzossie FE, Tsabang N, Nkongmeneck BA, Nguenang GM, Auzel P, Christina E, Kamou E, Balouma JM, Apalo P, Mathieu H, Valbuena M, Valère M. 2008. Les peuplements d'arbres du Sanctuaire à gorilles de Mengamé au sud Camreoun. Tropical Conservation Science, 1(3): 204221.

Grisvard P, Chaudun V, Chouard P, Guillaumin A, Schneiter P. 1990. Le Bon Jardinier Encyclopédie Horticole $\left(152^{\mathrm{e}}\right.$ édn). Tome I. La Maison Rustique : Paris ; $883 \mathrm{p}$.

Grisvard P, Chaudun V, Chouard P, Guillaumin A, Schneiter P. 1990. Le Bon 
Jardinier Encyclopédie Horticole $\left(152^{\mathrm{e}}\right.$ édn). Tome II. La Maison Rustique : Paris ; $783 \mathrm{p}$.

Guelly KA. 1994. Les savanes de la zone forestière subhumide du Togo. Th. Doct. Univ. Pierre et Marie Curie, Paris VI, 163 p.

Lebrun J-P, Stork A. 2003. Tropical African Flowering Plants. Ecology and Distribution. Vol. 1: AnnonaceaeBalanitaceae. Conservatoire et Jardin Botaniques de la Ville de Genève. Hors série $9 ; 793$.

Lebrun J-P, Stork A. 2006. Tropical African Flowering Plants. Ecology and Distribution. Vol. 2: EuphorbiaceaeDichapetalaceae. Conservatoire et Jardin Botaniques de la Ville de Genève. Hors série $9 \mathrm{a} ; 306$.

Lebrun J.-P, Stork A. 2008. Tropical African Flowering Plants. Ecology and Distribution. Vol. 3: MimosaceaeFabaceae. Conservatoire et Jardin Botaniques de la Ville de Genève. Hors série ; 325.

Lebrun J-P, Stork A. 2008. Tropical African

Flowering Plants. Ecology and Distribution. Vol. 4: Fabaceae (Desmodium-Zornia). Conservatoire et Jardin Botaniques de la Ville de Genève. Hors série ; 291.

Hessayon DG. 1992. The New House Plant Expert. PBI Publications : Herts ; 256 p.
Houerou (Le) V, Houerou (Le) J-Y. 1987. L'Arbre du Voyageur. Les Nouvelles Editions Africaines : Abidjan ; 186.

Hutchinson J, Dalziel JM. 1954-1972. Flora of West Tropical Africa (2nd edn revised). Vol. I-III. Keay RWJ \& Hepper FN: London.

Jaffee S, Masakure O. 2005. Strategic use of private standards to enhance international competitiveness: Vegetable exports from Kenya and elsewhere. Food Policy, 30 : 316-333.

Kokou K. 1998. Les mosaïques forestières au sud du Togo : biodiversité, dynamique et activités humaines. Th. Doct. Univ. Montpellier II, p. 140.

Maki K, Galatowitsch 2004. Movement of invasive aquatic plants into Minnesota (USA) through horticultural trade. Biological Conservation, 118 : 389-396.

Neven D, Odera MM, Rardon T, Wang H. 2009. Kenyan Supermarkets, Emerging Middle-Class Horticultural Farmers, and Employment Impacts on the Rural Poor. 2009. World Development, 37(11): 18021811.

Radji R. 1998. La flore horticole du Togo. J. Bot. Soc. Bot. France, 8: 87-94.

Roy D, Thorat A. 2008. Success in High Value Horticultural Export Markets for the Small Farmers: The Case of Mahagrapes in India. World Development, 36(10): 1874-1890. 